\title{
A STRONG COMPARISON THEOREM FOR SELFADJOINT ELLIPTIC EQUATIONS
}

\section{KURT KREITH}

The purpose of this note is to give a concise proof of a comparison theorem for selfadjoint, second order elliptic equations which yields stronger results than those previously derived in [1], [2] and [3]. All coefficients and domains are to be sufficiently smooth so that the variational techniques of Courant [4] can be applied. Specifically, it is assumed that the first eigenfunction of the selfadjoint boundary value problem

$$
\begin{gathered}
-\sum_{i, j=1}^{n} \frac{\partial}{\partial x_{j}}\left(\alpha_{i j} \frac{\partial v}{\partial x_{i}}\right)+\gamma v=\lambda v \quad \text { in } D, \\
\frac{\partial v}{\partial \nu}+\sigma v=0 \quad \text { on } \partial D, \quad-\infty<\sigma(x) \leqq+\infty
\end{gathered}
$$

can be determined uniquely (up to a multiplicative constant) by minimizing

$$
D[\phi]=\int_{D}\left[\sum \alpha_{i j} \frac{\partial \phi}{\partial x_{i}} \frac{\partial \phi}{\partial x_{j}}+\gamma \phi^{2}\right] d x+\int_{\partial D} \sigma \phi^{2} d \bar{x}
$$

over all "admissible" $\phi \in \Phi$. The class $\Phi$ consists of all real valued functions which are continuous in $\bar{D}$ have piecewise continuous first partials in $D$, vanish on $\{x \in \partial D \mid \sigma(x)=+\infty\}$ and satisfy $\int_{D} \phi^{2} d x=1$. (Here, $\sigma(x)=+\infty$ is used to denote the boundary condition $v=0$.) It is further assumed that all coefficients and $D$ are sufficiently regular so that this extremal function is a solution of (1) in the classical sense.

Theorem. Suppose $u(x)$ and $v(x)$ are solutions respectively of

$$
\begin{aligned}
& \sum \frac{\partial}{\partial x_{j}}\left(a_{i j} \frac{\partial u}{\partial x_{i}}\right)=c u, \\
& \sum \frac{\partial}{\partial x_{j}}\left(\alpha_{i j} \frac{\partial v}{\partial x_{i}}\right)=\gamma v
\end{aligned}
$$

in a domain $G \supset \bar{D}$ and that

Received by the editors March 10, 1967. 


$$
\int_{D}\left[\sum a_{i j} \frac{\partial u}{\partial x_{i}} \frac{\partial u}{\partial x_{j}}+c u^{2}\right] d x \geqq \int_{D}\left[\sum \alpha_{i j} \frac{\partial u}{\partial x_{i}} \frac{\partial u}{\partial x_{j}}+\gamma u^{2}\right] d x .
$$

If

$$
\partial u / \partial \nu+s(x) u=0 \quad \text { on } \partial D
$$

and

$$
\partial v / \partial \nu+\sigma(x) v=0 \quad \text { on } \partial D
$$

with $-\infty<\sigma(x) \leqq s(x) \leqq+\infty$, then either $v(x)$ has a zero in the interior of $D$ or else $u$ is a constant multiple of $v$.

Proof. Let $B_{1}=\{x \in \partial D \mid \sigma(x)<\infty\}$ and $B_{2}=\{x \in \partial D \mid s(x)<\infty\}$. Without loss of generality we may assume $\int_{D} u^{2} d x=1$ so that $u$ is admissible with respect to the variational problem (2). If $v(x) \neq 0$ in the interior of $D$, then $v$ is the first eigenfunction of (1) corresponding to the eigenvalue $\lambda_{1}=0$. Therefore

$$
\begin{aligned}
0 & =\inf _{\phi \in \Phi} \int_{D}\left[\sum \alpha_{i j} \frac{\partial \phi}{\partial x_{i}} \frac{\partial \phi}{\partial x_{j}}+\gamma \phi^{2}\right] d x+\int_{B_{1}} \sigma \phi^{2} d \bar{x} \\
& \leqq \int_{D}\left[\sum \alpha_{i j} \frac{\partial u}{\partial x_{i}} \frac{\partial u}{\partial x_{j}}+\gamma u^{2}\right] d x+\int_{B_{1}} \sigma u^{2} d \bar{x} \\
& \leqq \int_{D}\left[\sum a_{i j} \frac{\partial u}{\partial x_{i}} \frac{\partial u}{\partial x_{j}}+c u^{2}\right] d x+\int_{B_{2}} s u^{2} d \bar{x} .
\end{aligned}
$$

However in view of (3), (5) and Green's theorem, the last term is zero so that we have equality throughout the above expression. In particular, we see that $u(x)$ is an extremal function for the variational problem (2) and therefore an eigenfunction of (1) corresponding to $\lambda_{1}=0$. In light of the simplicity of the first eigenvalue of (1), $u$ is a constant multiple of $v$.

Setting $s(x) \equiv+\infty$ on $\partial D$, we obtain a stronger form of the comparison theorem derived in [3].

\section{BIBLIOGRAPHY}

1. P. Hartman and A. Wintner, On a comparison theorem for self-adjoint partial differential equations of elliptic type, Proc. Amer. Math. Soc. 6 (1955), 862-865.

2. K. Kreith, $A$ new proof of a comparison theorem for elliptic equations, Proc. Amer. Math. Soc. 14 (1963), 33-35.

3. C. Clark and C. A. Swanson, Comparison theorems for elliptic differential equations, Proc. Amer. Math. Soc. 16 (1965), 886-890.

4. R. Courant, Über die Eigenwerte bei den Differentialgleichungen der mathematischen Physik, Math. Z. 7 (1920), 1-57.

University of California, Davis 